\title{
Ramanujan-type Congruences for Broken 2-Diamond Partitions Modulo 3
}

\author{
William Y.C. Chen ${ }^{1}$, Anna R.B. Fan ${ }^{2}$ and Rebecca T. Yu ${ }^{3}$ \\ ${ }^{1,2,3}$ Center for Combinatorics, LPMC-TJKLC \\ Nankai University, Tianjin 300071, P. R. China \\ ${ }^{1}$ Center for Applied Mathematics \\ Tianjin University, Tianjin 300072, P. R. China \\ Email: ${ }^{1}$ chen@nankai.edu.cn, ${ }^{2}$ fanruice@mail.nankai.edu.cn, \\ ${ }^{3}$ yuting_shuxue@mail.nankai.edu.cn
}

\begin{abstract}
The notion of broken $k$-diamond partitions was introduced by Andrews and Paule. Let $\Delta_{k}(n)$ denote the number of broken $k$-diamond partitions of $n$. They also posed three conjectures on the congruences of $\Delta_{2}(n)$ modulo 2,5 and 25 . Hirschhorn and sellers proved the conjectures for modulo 2, and Chan proved cases of modulo 5. For the case of modulo 3, Radu and Sellers obtained an infinite family of congruences for $\Delta_{2}(n)$. In this paper, we obtain two infinite families of congruences for $\Delta_{2}(n)$ modulo 3 based on a formula of Radu and Sellers, the 3-dissection formula of the generating function of triangular number due to Berndt, and the properties of the $U$-operator, the $V$-operator, the Hecke operator and the Hecke eigenform. For example, we find that $\Delta_{2}(243 n+142) \equiv \Delta_{2}(243 n+223) \equiv 0(\bmod 3)$. The infinite family of Radu and Sellers and the two infinite families derived in this paper have two congruences in common, namely, $\Delta_{2}(27 n+16) \equiv \Delta_{2}(27 n+25) \equiv 0(\bmod 3)$.
\end{abstract}

Keywords: broken $k$-diamond partition, modular form, Ramanujan-type congruence, Hecke eigenform

AMS Classification: 05A17, 11P83

\section{Introduction}

The objective of this paper is to derive two infinite families of congruences for the number of broken 2-diamond partitions modulo 3. The notion of broken $k$-diamond partitions was introduced by Andrews and Paule [1]. A broken $k$-diamond partition $\pi=\left(a_{1}, a_{2}, a_{3}, \ldots ; b_{2}, b_{3}, b_{4}, \ldots\right)$ is a plane partition satisfying the relations illustrated 
in Figure 1.1, where $a_{i}, b_{i}$ are nonnegative integers and $a_{i} \rightarrow a_{j}$ means $a_{i} \geq a_{j}$. More precisely, each building block in Figure 1.1, except for the broken block $\left(b_{2}, b_{3}, \ldots b_{2 k+2}\right)$ has the same order structure as shown in Figure 1.2. We call each block a $k$-elongated partition diamond of length 1 , or a $k$-elongated diamond, for short.

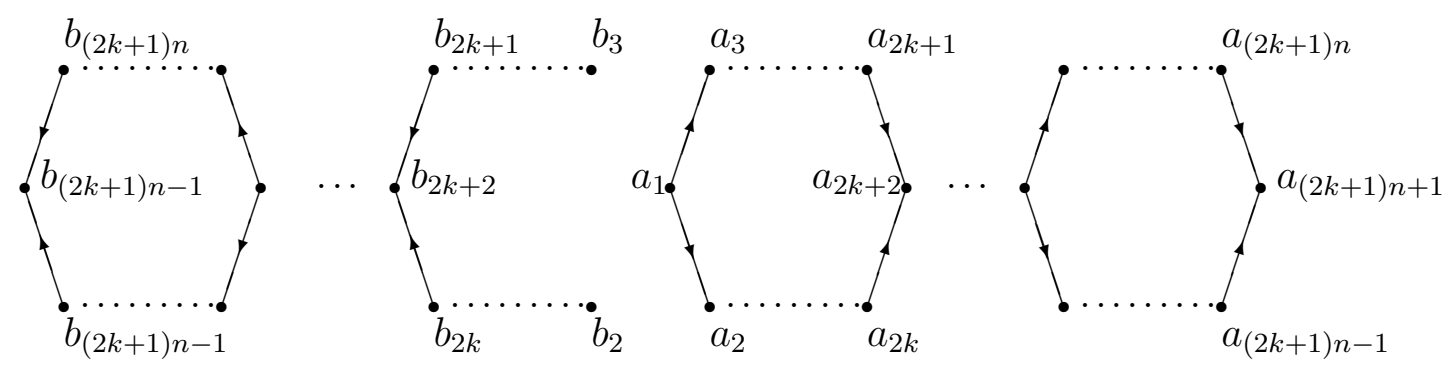

Figure 1.1: A broken $k$-diamond of length $2 n$.

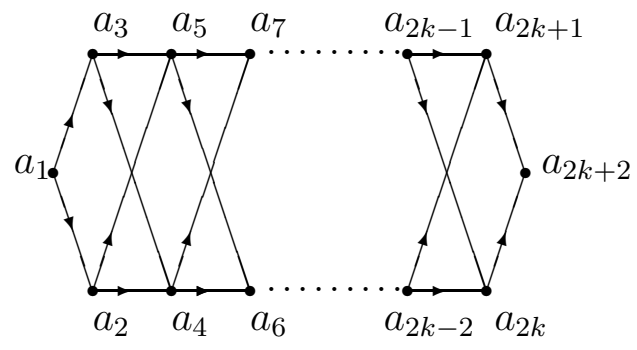

Figure 1.2: A $k$-elongated diamond.

For example, Figure 1.3 gives a broken 2-diamond partition

$$
\pi=(10,8,9,7,6,3,2,1,0,1 ; 3,5,2,1,1,1) .
$$

Let $\Delta_{k}(n)$ denote the number of broken $k$-diamond partitions of $n$, and let $B_{k}(q)$ denote the generating function for $\Delta_{k}(n)$, that is

$$
B_{k}(q)=\sum_{n \geq 0} \Delta_{k}(n) q^{n} .
$$

Andrews and Paule [1] obtained the following formula:

$$
B_{k}(q)=\frac{(-q ; q)_{\infty}}{(q ; q)_{\infty}^{2}\left(-q^{2 k+1} ; q^{2 k+1}\right)_{\infty}} .
$$



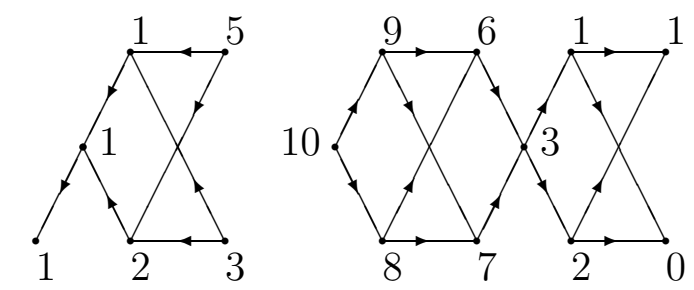

Figure 1.3: A broken 2-diamond partition of 60 .

Note that the above formula can also be written in terms of eta-quotients related to modular forms:

$$
B_{k}(q)=q^{(k+1) / 12} \frac{\eta(2 z) \eta((2 k+1) z)}{\eta(z)^{3} \eta((4 k+2) z)}
$$

where $q=e^{2 \pi i z}$.

From (1.1), Andrews and Paule proved that for $n \geq 3$,

$$
\Delta_{1}(2 n+1) \equiv 0 \quad(\bmod 3)
$$

They also posed three conjectures on congruences for the number of broken 2-diamond partitions of $n . \mathrm{Fu}$ [5] and Mortenson [8] found combinatorial proofs of congruence (1.2). Meanwhile, Hirschhorn and Sellers [6] gave a proof of (1.2) by deriving the following generating formula for $\Delta_{1}(2 n+1)$

$$
\sum_{n \geq 0} \Delta_{1}(2 n+1) q^{n}=3 \frac{\left(q^{2} ; q^{2}\right)_{\infty}^{2}\left(q^{6} ; q^{6}\right)_{\infty}^{2}}{(q ; q)_{\infty}^{6}}
$$

Hirschhorn and Sellers also obtained the following congruences modulo 2

$$
\begin{aligned}
& \Delta_{1}(4 n+2) \equiv \Delta_{1}(4 n+3) \equiv 0 \quad(\bmod 2), \\
& \Delta_{2}(10 n+2) \equiv \Delta_{2}(10 n+6) \equiv 0 \quad(\bmod 2),
\end{aligned}
$$

where $n \geq 1$. The congruences in (1.4) were conjectured by Andrews and Paule [1].

Chan [4] found the following two infinite families of congruences modulo 5 for broken 2-diamond partitions:

$$
\begin{aligned}
& \Delta_{2}\left(5^{l+1}+\frac{3}{4}\left(5^{l}-1\right)+2 \cdot 5^{l}+1\right) \equiv 0 \quad(\bmod 5), \\
& \Delta_{2}\left(5^{l+1}+\frac{3}{4}\left(5^{l}-1\right)+4 \cdot 5^{l}+1\right) \equiv 0 \quad(\bmod 5),
\end{aligned}
$$


where $l \geq 1$. The cases for $l=1$ in (1.5) and (1.6) were conjectured by Andrews and Paule [1], namely,

$$
\Delta_{2}(25 n+14) \equiv \Delta_{2}(25 n+24) \equiv 0 \quad(\bmod 5)
$$

Paule and Radu [10] obtained the following infinite family of congruences modulo 5 for broken 2-diamond partitions. They showed that for any prime $p$ with $p \equiv 13,17$ $(\bmod 20)$ and any nonnegative integer $n$,

$$
\Delta_{2}\left(5 p^{2} n+4 p-\frac{1}{4}(p-1)\right) \equiv 0 \quad(\bmod 5) .
$$

Moreover, they posed four conjectures on congruences for broken 3-diamond partitions and broken 5-diamond partitions, which have been confirmed by Xiong [12] and Jameson [7].

For the broken 2-diamond partitions, Radu and Sellers [11] showed that

$$
\sum_{n \geq 0} \Delta_{2}(3 n+1) q^{n} \equiv 2 q \prod_{n \geq 1} \frac{\left(1-q^{10 n}\right)^{4}}{\left(1-q^{5 n}\right)^{2}} \quad(\bmod 3),
$$

which implies the following congruence relations

$$
\begin{aligned}
& \Delta_{2}(15 n+1) \equiv \Delta_{2}(15 n+7) \equiv 0 \quad(\bmod 3), \\
& \Delta_{2}(15 n+10) \equiv \Delta_{2}(15 n+13) \equiv 0 \quad(\bmod 3),
\end{aligned}
$$

and

$$
\Delta_{2}\left(3 p^{2} n+\frac{3}{4}(p(4 k+3)-1)+1\right) \equiv 0 \quad(\bmod 3),
$$

where prime $p \equiv 3(\bmod 4), 0 \leq k \leq p-1$ and $k \neq \frac{p-3}{4}$.

In this paper, we use (1.8) to establish two new infinite families of congruences of $\Delta_{2}(n)$ modulo 3 by using the 3-dissection formula of the generating function of triangular numbers and properties of the $U$-operator, the $V$-operator, the Hecke operator and the Hecke eigenform.

Theorem 1.1 For $l \geq 1$, we have

$$
\begin{aligned}
& \Delta_{2}\left(3^{2 l+1} n+\frac{3}{4}\left(3^{2 l}-1\right)+3^{2 l}+1\right) \equiv 0 \quad(\bmod 3) \\
& \Delta_{2}\left(3^{2 l+1} n+\frac{3}{4}\left(3^{2 l}-1\right)+2 \cdot 3^{2 l}+1\right) \equiv 0 \quad(\bmod 3) .
\end{aligned}
$$




\section{Preliminaries}

In this section, we give an overview of some definitions and properties of modular forms which will be used in the proof of Theorem 1.1. For more details on the theory of modular forms, see for example, Ono [9]. In this paper, we shall be concerned with the modular forms for the congruence subgroup $\Gamma_{0}(N)$ of $S L_{2}(\mathbb{Z})$, where

$$
\Gamma_{0}(N):=\left\{\left(\begin{array}{ll}
a & b \\
c & d
\end{array}\right) \in S L_{2}(\mathbb{Z}) \mid c \equiv 0 \quad(\bmod N)\right\} .
$$

First, recall the definition of a modular form.

Definition 2.1 Let $k$ be a positive integer and $\chi$ be a Dirichlet character modulo $N$, we say that a meromorphic function $f(z): \mathbb{H} \rightarrow \mathbb{C}$ is a modular form for $\Gamma_{0}(N)$ with Nebentypus $\chi$ and weight $k$, if

(1) $f(z)$ is a holomorphic function on $\mathbb{H}$.

(2) $f(z)$ satisfies the following relation for $z \in \mathbb{H}$ and $\gamma \in \Gamma_{0}(N)$

$$
f(\gamma z)=\chi(d)(c z+d)^{k} f(z) .
$$

(3) $f(z)$ is holomorphic at the cusps of $\Gamma_{0}(N)$, here the cusps are the equivalent classes of $Q \cup\{\infty\}$ on the action of $\Gamma_{0}(N)$.

The set of modular forms for $\Gamma_{0}(N)$ with Nebentypus $\chi$ and weight $k$ is denoted by $M_{k}\left(\Gamma_{0}(N), \chi\right)$. When $\chi$ is trivial, that is, $\chi(d)=1$ for all integers $d$, we denote $M_{k}\left(\Gamma_{0}(N), \chi\right)$ by $M_{k}\left(\Gamma_{0}(N)\right)$. In addition, if $f(z)$ vanishes at the cusps of $\Gamma_{0}(N)$, then we say that $f(z)$ is a cusp form. We denote by $S_{k}\left(\Gamma_{0}(N), \chi\right)$ the set of cusp forms for $\Gamma_{0}(N)$ with Nebentypus $\chi$ and weight $k$.

To prove Theorem 1.1, we need the operators $U, V$ and the Hecke operator.

Definition 2.2 Let $f(z)=\sum_{n \geq 0} a(n) q^{n}$ be a modular form in $M_{k}\left(\Gamma_{0}(N), \chi\right)$. If $d$ is a positive integer, then the $U$-operator $U(d)$ and the $V$-operator $V(d)$ are defined by

$$
f(z) \mid U(d):=\sum_{n \geq 0} a(d n) q^{n}
$$

and

$$
f(z) \mid V(d):=\sum_{n \geq 0} a(n) q^{d n}
$$

For any positive integer $m$, the action of the $m$-th Hecke operator $T_{m, k, \chi}:=T(m)$ is given by

$$
f(z) \mid T(m):=\sum_{n \geq 0}\left(\sum_{d \mid \operatorname{gcd}(m, n)} \chi(d) d^{k-1} a\left(m n / d^{2}\right)\right) q^{n}
$$


In particular, for any prime $p$

$$
f(z) \mid T(p):=\sum_{n \geq 0}\left(a(p n)+\chi(p) p^{k-1} a(n / p)\right) q^{n} .
$$

From the definition of the $U$-operator and the Hecke operator, one sees that for any prime $p$, we have

$$
f(z)|U(p) \equiv f(z)| T(p) \quad(\bmod p) .
$$

The operators $U, V$ and the Hecke operator have the following properties.

Proposition 2.3 Suppose that $f(z) \in M_{k}\left(\Gamma_{0}(N), \chi\right)$.

(1) If $d$ is a positive integer, then

$$
f(z) \mid V(d) \in M_{k}\left(\Gamma_{0}(N d), \chi\right) .
$$

(2) If $d \mid N$, then

$$
f(z) \mid U(d) \in M_{k}\left(\Gamma_{0}(N), \chi\right) .
$$

(3) If $m$ is a positive integer, then

$$
f(z) \mid T(m) \in M_{k}\left(\Gamma_{0}(N), \chi\right) .
$$

A Hecke eigenform associated with the Hecke operators is defined as follows.

Definition 2.4 A modular form $f(z) \in M_{k}\left(\Gamma_{0}(N), \chi\right)$ is called a Hecke eigenform if for every $m \geq 2$ there is a complex number $\lambda(m)$ for which

$$
f(z) \mid T(m)=\lambda(m) f(z) .
$$

If a Hecke eigenform is a cusp form, then the following proposition can be used to compute the value $\lambda(m)$.

Proposition 2.5 Suppose that $f(z)=\sum_{n \geq 0} a(n) q^{n}$ is a cusp form in $S_{k}\left(\Gamma_{0}(N), \chi\right)$ with $a(1)=1$. If $f(z)$ is an eigenform, then for $m \geq 1$,

$$
f(z) \mid T(m)=a(m) f(z) .
$$




\section{Proof of the Main Theorem}

In this section, we give a proof of Theorem 1.1 by using the approach of Chan [4]. We first establish the following congruence on the generating function of $\Delta_{2}(3 n+1)$. Let $\psi(q)$ be the generating function for the triangular numbers, that is,

$$
\psi(q)=\sum_{n \geq 0} q^{n(n+1) / 2}=\prod_{n \geq 1} \frac{\left(1-q^{2 n}\right)^{2}}{\left(1-q^{n}\right)} .
$$

Lemma 3.1 We have

$$
\psi\left(q^{15}\right)^{2} \sum_{n \geq 0} \Delta_{2}(3 n+1) q^{n+4} \equiv 2 q^{5} \psi\left(q^{5}\right)^{8} \quad(\bmod 3) .
$$

Proof. By (1.8) and (3.1), we see that

$$
\sum_{n \geq 0} \Delta_{2}(3 n+1) q^{n} \equiv 2 q \prod_{n \geq 1} \frac{\left(1-q^{10 n}\right)^{4}}{\left(1-q^{5 n}\right)^{2}}=2 q \psi\left(q^{5}\right)^{2} \quad(\bmod 3) .
$$

It follows that

$$
\begin{aligned}
2 q^{5} \psi\left(q^{5}\right)^{8} & =q^{4} \psi\left(q^{5}\right)^{6} \cdot\left(2 q \psi\left(q^{5}\right)^{2}\right) \\
& \equiv \psi\left(q^{5}\right)^{6} \sum_{n \geq 0} \Delta_{2}(3 n+1) q^{n+4} \quad(\bmod 3) .
\end{aligned}
$$

Since

$$
\frac{(q ; q)_{\infty}^{3}}{\left(q^{3} ; q^{3}\right)_{\infty}} \equiv 1 \quad(\bmod 3)
$$

see, Ono [9], we find that

$$
\psi\left(q^{5}\right)^{6} \sum_{n \geq 0} \Delta_{2}(3 n+1) q^{n+4} \equiv \psi\left(q^{15}\right)^{2} \sum_{n \geq 0} \Delta_{2}(3 n+1) q^{n+4} \quad(\bmod 3) .
$$

Combining (3.4) and (3.5), we obtain that

$$
\psi\left(q^{15}\right)^{2} \sum_{n \geq 0} \Delta_{2}(3 n+1) q^{n+4} \equiv 2 q^{5} \psi\left(q^{5}\right)^{8} \quad(\bmod 3),
$$

as desired.

It is not difficult to show that $q^{5} \psi\left(q^{5}\right)^{8}$ is a Hecke eigenform.

Lemma 3.2 The function $q^{5} \psi\left(q^{5}\right)^{8}$ is a Hecke eigenform in $M_{4}\left(\Gamma_{0}(10)\right)$. More precisely, for $m \geq 2$, we have

$$
q^{5} \psi\left(q^{5}\right)^{8} \mid T(m)=\lambda(m) q^{5} \psi\left(q^{5}\right)^{8},
$$

where $\lambda(m)=\left[q^{m}\right] q \psi(q)^{8}$, that is, the coefficient of $q^{m}$ in $q \psi(q)^{8}$. 
Proof. It has been shown by Chan [3] that $q \psi(q)^{8}$ is a Hecke eigenform in $M_{4}\left(\Gamma_{0}(2)\right)$. By Definition 2.4, for $m \geq 2$, there exits a complex number $\lambda(m)$ such that

$$
q \psi(q)^{8} \mid T(m)=\lambda(m) q \psi(q)^{8} .
$$

Observing that $q \psi(q)^{8}$ is a cusp form for which the coefficient of $q$ is 1 , by Proposition 2.5 we find

$$
\lambda(m)=\left[q^{m}\right] q \psi(q)^{8} .
$$

Substituting $q$ by $q^{5}$ in (3.6), we obtain that

$$
q^{5} \psi\left(q^{5}\right)^{8} \mid T(m)=\lambda(m) q^{5} \psi\left(q^{5}\right)^{8} .
$$

Meanwhile, by Proposition 2.3 (2), we have

$$
q^{5} \psi\left(q^{5}\right)^{8}=q \psi(q)^{8} \mid V(5) \in M_{4}\left(\Gamma_{0}(10)\right) .
$$

Thus $q^{5} \psi\left(q^{5}\right)^{8}$ is a Hecke eigenform in $M_{4}\left(\Gamma_{0}(10)\right)$.

We are now ready to prove Theorem 1.1 .

Proof of Theorem 1.1. Let $f(z)=2 q^{5} \psi\left(q^{5}\right)^{8}$ with $q=e^{2 \pi i z}$. Applying the $U$-operator $U(3)$ to $f(z)$ gives

$$
\begin{aligned}
2 q^{5} \psi\left(q^{5}\right)^{8} \mid U(3) & \equiv\left(\psi\left(q^{15}\right)^{2} \sum_{n \geq 0} \Delta_{2}(3 n+1) q^{n+4}\right) \mid U(3) \quad(\bmod 3) \\
& =\psi\left(q^{5}\right)^{2} \sum_{n \geq 0} \Delta_{2}(9 n-11) q^{n}
\end{aligned}
$$

On the other hand, Lemma 3.2 implies that

$$
\begin{aligned}
q^{5} \psi\left(q^{5}\right)^{8} \mid T(3) & =\left(\left[q^{3}\right] q \psi(q)^{8}\right) q^{5} \psi\left(q^{5}\right)^{8} \\
& =28 q^{5} \psi\left(q^{5}\right)^{8} \equiv q^{5} \psi\left(q^{5}\right)^{8} \quad(\bmod 3) .
\end{aligned}
$$

Employing relation (2.4), we deduce that

$$
\begin{aligned}
\psi\left(q^{5}\right)^{2} \sum_{n \geq 0} \Delta_{2}(9 n-11) q^{n} & \equiv 2 q^{5} \psi\left(q^{5}\right)^{8} \mid U(3) \\
& \equiv 2 q^{5} \psi\left(q^{5}\right)^{8} \mid T(3) \equiv 2 q^{5} \psi\left(q^{5}\right)^{8}
\end{aligned}
$$

Substituting $n$ by $n+2$ in the above congruence, we get

$$
\sum_{n \geq 0} \Delta_{2}(9 n+7) q^{n} \equiv 2 q^{3} \psi\left(q^{5}\right)^{6} \equiv 2 q^{3} \psi\left(q^{15}\right)^{2} \quad(\bmod 3) .
$$

By the 3-dissection formula of $\psi(q)$ due to Berndt [2, p49], one sees that $\psi(q)$ can be written in the following form

$$
\psi(q)=A\left(q^{3}\right)+q \psi\left(q^{9}\right),
$$


where $A(q)$ is power series in $q$. Plugging (3.9) into the right hand side of (3.8), we find

$$
\begin{aligned}
\sum_{n \geq 0} \Delta_{2}(9 n+7) q^{n} & \equiv 2 q^{3}\left(A\left(q^{45}\right)+q^{15} \psi\left(q^{135}\right)\right)^{2} & (\bmod 3) \\
& \equiv 2 q^{3}\left(A\left(q^{45}\right)^{2}+2 q^{15} A\left(q^{45}\right) \psi\left(q^{135}\right)+q^{30} \psi\left(q^{135}\right)^{2}\right) & (\bmod 3) .
\end{aligned}
$$

Extracting those terms whose powers of $q$ are congruent to 6 modulo 9 in (3.10), we have

$$
\sum_{n \geq 0} \Delta_{2}(9(9 n+6)+7) q^{9 n+6} \equiv 2 q^{33} \psi\left(q^{135}\right)^{2} \quad(\bmod 3) .
$$

Dividing both sides of (3.11) by $q^{6}$ and replacing $q^{9}$ by $q$, we deduce that

$$
\sum_{n \geq 0} \Delta_{2}\left(3^{4} n+61\right) q^{n} \equiv 2 q^{3} \psi\left(q^{15}\right)^{2} \quad(\bmod 3) .
$$

Combining (3.8) and (3.12), we arrive at

$$
\sum_{n \geq 0} \Delta_{2}\left(3^{2} n+7\right) q^{n} \equiv \sum_{n \geq 0} \Delta_{2}\left(3^{4} n+61\right) q^{n} \quad(\bmod 3) .
$$

Iterating (3.13) with $n$ replaced by $9 n+6$, we conclude that for $l \geq 1$,

$$
\sum_{n \geq 0} \Delta_{2}\left(3^{2} n+7\right) q^{n} \equiv \sum_{n \geq 0} \Delta_{2}\left(3^{2 l} n+\frac{3}{4}\left(3^{2 l}-1\right)+1\right) q^{n} \quad(\bmod 3) .
$$

From (3.8) together with the above relation, it follows that

$$
\sum_{n \geq 0} \Delta_{2}\left(3^{2 l} n+\frac{3}{4}\left(3^{2 l}-1\right)+1\right) q^{n} \equiv 2 q^{3} \psi\left(q^{15}\right)^{2} \quad(\bmod 3) .
$$

Since there are no terms with powers of $q$ congruent to 1,2 modulo 3 in $2 q^{3} \psi\left(q^{15}\right)^{2}$, substituting $n$ by $3 n+1$ and $3 n+2$ respectively in (3.14), we obtain the following infinite families of congruences

$$
\begin{aligned}
\Delta_{2}\left(3^{2 l+1} n+\frac{3}{4}\left(3^{2 l}-1\right)+3^{2 l}+1\right) & \equiv 0 \quad(\bmod 3), \\
\Delta_{2}\left(3^{2 l+1} n+\frac{3}{4}\left(3^{2 l}-1\right)+2 \cdot 3^{2 l}+1\right) & \equiv 0 \quad(\bmod 3) .
\end{aligned}
$$

This completes the proof.

Here are some examples of Theorem 1.1, For $n \geq 0$, we have

$$
\begin{aligned}
& \Delta_{2}(27 n+16) \equiv \Delta_{2}(27 n+25) \equiv 0 \quad(\bmod 3) \\
& \Delta_{2}(243 n+142) \equiv \Delta_{2}(243 n+223) \equiv 0 \quad(\bmod 3) \\
& \Delta_{2}(2187 n+1276) \equiv \Delta_{2}(2187 n+2005) \equiv 0 \quad(\bmod 3) .
\end{aligned}
$$


Notice that congruences in (3.15) are also contained in the infinite family of congruences (1.11) derived by Radu and Sellers.

Acknowledgments. This work was supported by the 973 Project, the PCSIRT Project of the Ministry of Education, and the National Science Foundation of China.

\section{References}

[1] G.E. Andrews and P. Paule, Macmahon's partition analysis XI: broken diamonds and modular forms, Acta Arith., 126 (2007), 281-294.

[2] B.C. Berndt, Ramanujan's Notebooks, Part III, Springer-Verlag, New York, 1991.

[3] H.H. Chan, S. Cooper and W.-C Liaw, An odd square as a sum of an odd number of odd squares, Acta Arith., 132 (2008), 359-371.

[4] S.H. Chan, Some congruences for Andrews-Paule's broken 2-diamond partitions, Discrete Math., 308 (2008), 5735-5741.

[5] S.S. Fu, Combinatorial proof of one congruence for the broken 1-diamond partition and a generalization, Int. J. Number Theory, 7 (2011), 133-144.

[6] M.D. Hirschhorn and J.A. Sellers, On recent congruence results of Andrews and Paule for broken $k$-diamonds, Bull. Austral. Math. Soc., 75 (2007), 121-126.

[7] M. Jameson, Congruences for broken $k$-diamond partitions, Ann. Combin., 17 (2013), to appear.

[8] E. Mortenson, On the broken 1-diamond partition, Int. J. Number Theory, 4 (2008), 199-218.

[9] K. Ono, The Web of Modularity: Arithmetic of the Coefficients of Modular Forms and q-series, CBMS Regional Conference Series in Mathematics, 102, Amer. Math. Soc., Province, RI, 2004.

[10] P. Paule and S. Radu, Infinite families of strange partition congruences for broken 2-diamonds, Ramanujan J., 23 (2010), 409-416.

[11] S. Radu and J.A. Sellers, Infinitely many congruences for broken 2-diamond partitions modulo 3, J. Combin. Number Theory, to appear.

[12] X.H. Xiong, Two congruences involving Andrews-Paule's broken 3-diamond partitions and 5-diamond partitions, Proc. Japan Acad. Ser. A Math. Sci., 87 (2011), 65-68. 